\title{
Structured home-based exercise program for improving walking ability in ambulant children with cerebral palsy
}

\author{
Aishah Ahmad Fauzi ${ }^{\mathrm{a}, *}$, Masyitah Mohammad Khayat ${ }^{\mathrm{a}}$, Sakinah Sabirin ${ }^{\mathrm{a}}$, Norazah Haron ${ }^{\mathrm{b}}$, \\ Mohd Nahar Azmi Mohamed ${ }^{\mathrm{C}}$ and Glen M. Davis ${ }^{\mathrm{d}}$ \\ ${ }^{a}$ Department of Rehabilitation Medicine, Faculty of Medicine, University Malaya, Kuala Lumpur, Malaysia \\ ${ }^{\mathrm{b}}$ Department of Rehabilitation Medicine, University Malaya Medical Centre, Kuala Lumpur, Malaysia \\ ${ }^{\mathrm{c}}$ Department of Sports Medicine, Faculty of Medicine, University Malaya, Kuala Lumpur, Malaysia \\ ${ }^{\mathrm{d}}$ Clinical Exercise and Rehabilitation Unit, Faculty of Health Sciences, The University of Sydney, Sydney, NSW, \\ Australia
}

\begin{abstract}
.
OBJECTIVE: To investigate outcomes after 8 weeks of a structured home-based exercise program (SHEP) for improving walking ability in ambulant children with cerebral palsy (CP).

METHOD: Eleven children participated in this study ( 7 males and 4 females, mean age 10 years 3 months, standard deviation (SD) 3y) with Gross Motor Function Classification System (GMFCS) I-III. This study used a prospective multiple assessment baseline design to assess the effect of SHEP upon multiple outcomes obtained in three different phases. Exercise intensity was quantified by OMNI-RPE assessed by caregivers and children. Outcome assessments of walking speed, GMFM-66 and physiological cost index (PCI) were measured four times at pre-intervention (Phase 1) and at 3-weekly intervals over eight weeks during intervention (Phase 2). Follow-up assessments were performed at one month and three months after intervention (Phase 3). Statistical analyses were repeated measures ANOVA and Wilcoxon signed-rank test.

RESULTS: SHEP improved walking ability in children with CP, particularly for their walking speed ( $p=0.01$, Cohen's $d=$ 1.9). The improvement of GMFM-66 scores during Phase 2 and Phase 3 had a large effect size, with Cohen's $d$ of 1.039 and 1.054 , respectively, compared with that during Phase $1(p<0.017)$. No significant change of PCI was observed (Cohen's $d=$ 0.39 ).

CONCLUSION: SHEP can be a useful intervention tool, given as a written, structured, and practical exercise program undertaken at home to achieve short term goals for improving walking ability when added to standard care.
\end{abstract}

Keywords: Home-based, exercise, ambulant, cerebral palsy, walking

\section{Introduction}

Cerebral palsy $(\mathrm{CP})$ is one of the most common causes of disability in children, requiring ongoing rehabilitation [1]. CP is a neurological disorder associ-

\footnotetext{
*Corresponding author: Aishah Ahmad Fauzi, Department of Rehabilitation Medicine, Faculty of Medicine, University Malaya, 50603 Kuala Lumpur, Malaysia. Tel.: +60 133417 577; E-mail: aishahaf@ummc.edu.my.
}

ated with movement and postural disability and is attributed to nonprogressive disturbances in the developing foetal or infant brain [2]. Although the pathophysiology of CP is nonprogressive, the progression of musculoskeletal deformities during a child's growth may deteriorate physical function and quality of life over time [3].

The rehabilitation of children with $\mathrm{CP}$ through approaches based on physical activity and exercise should maximise physical functioning, prevent sec- 
ondary musculoskeletal impairments and foster the cognitive, social and emotional development of the child [4]. Some structured exercise programs have planned or predetermined guidelines regarding the types of physical activities and the components of exercise prescription for the participant, whereas unstructured activity refers to any physical activity without any specific rules or exercise prescription [5]. Verschuren et al. demonstrated that an eight month structured exercise program incorporating aerobic and anaerobic circuits performed twice weekly in 45 minute sessions provided results with significant training effects on improvement of aerobic capacity, agility and muscle strength, as well as significant improvement in the participation level and quality of life of children and adolescents with CP [6].

In standard care, clinicians should prescribe various physical activity based interventions for children with $\mathrm{CP}$ on the basis of currently available evidence [7]. However, children with $\mathrm{CP}$ and their caregivers face numerous obstacles to adopt physical activity programs within usual care. These obstacles include personal, social and environmental barriers, as well as financial or time constraints. Moreover, logistical issues and the availability of therapists hinder children with $\mathrm{CP}$ from committing to a prescribed exercise programme $[8,9]$.

Home-based interventions may have some distinct advantages to overcome these barriers for children with $\mathrm{CP}$ to undertake exercise or physical activity programmes. Implementing physical activity or exercises at home complements the standard face-toface therapy with therapist to ensure continuity of therapy and achieve a certain level of intensity for a desired outcome $[10,11]$. The potential of several types of home-based exercise programme for children with CP has been explored; such types include body weight-supported treadmill exercise, server-based interactive training systems and an individualised homebased fitness training programme [12-14]. The oncea-week individualised home-based fitness training can improve physical fitness and muscle strength [12]. Moreover, home-based body weight-supported treadmill training exercise can improve function and gait even after completing the 12 week intervention period [13]. Improvements in daily activities, upper limb function and functional tests of lower limbs (sit to stand, lateral step up, half knee to standing) can be also remarkably improved after a 20 week home serverbased interactive training system [14]. Meanwhile, Van Wely et al. undertook a multicentre study involv- ing 49 walking children with spastic $\mathrm{CP}$ and demonstrated that the combination of home-based physiotherapy programmes with fitness training and counselling components positively improved the children's motor function (gross motor function measure-66 [GMFM66]) and walking ability (1 minute walk test) on the sixth and fourth month, respectively [15]. These observations had motivated and prompted rehabilitation physicians and physiotherapists of the paediatric rehabilitation unit in the University Malaya Medical Centre (UMMC) to develop a practical and structured homebased exercise programme (SHEP), which only requires low-cost equipment with appropriate exercise guidelines, a written guide, and a logbook. Hence, SHEP can be a practical, safe and convenient physical activity based intervention for children with CP.

The current study aimed to investigate the outcomes after the application of an 8 week SHEP for the improvement of the walking ability of ambulant children with $\mathrm{CP}$.

\section{Materials and methodology}

This study used a prospective multiple assessment baseline design to assess the effect of SHEP intervention by comparing multiple outcome measurements obtained in three different phases. Baselines were first established through repeated observations prior to the intervention, which was implemented at different times for different participants.

Prior to SHEP intervention, all participants and their caregivers attended familiarisation sessions with trained assessors who were physiotherapists from the Paediatric Rehabilitation Unit of UMMC, Kuala Lumpur, Malaysia. The baseline outcome was measured at weekly intervals over 4 weeks (pre-intervention, Phase 1). Next, it was measured three times over 8 weeks of the SHEP intervention (Phase 2). Followup assessments were conducted at 1 and 3 months after the intervention (Phase 3) (Fig. 1).

This study was approved by the Medical Ethics Committee of the UMMC (MECID. NO: 201581543), and all parents or main caregivers of children with $\mathrm{CP}$ (participants) provided a written informed consent.

\subsection{Participants}

Participants were recruited from the Paediatric Rehabilitation clinic registry over the period of 2013 to 2015. The inclusion criteria were as follows: children 

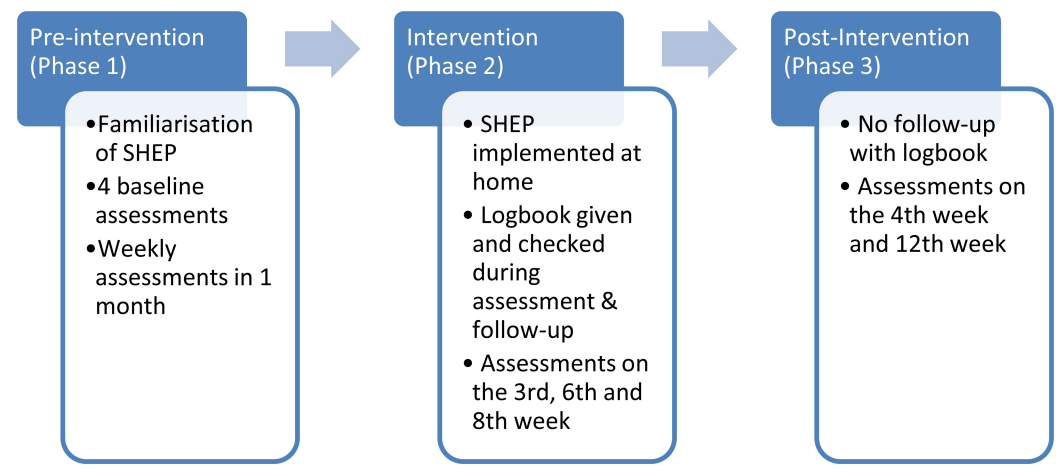

Fig. 1. Assessment phases.

aged 6-17 years, those who had CP and Gross Motor Function Classification System (GMFCS) I-III, those who could follow at least a single two step command through bedside clinical assessment by a rehabilitation physician, and those who received standard rehabilitation care. Meanwhile, the exclusion criteria were as follows: children with $\mathrm{CP}$ who were unable to comprehend a two-step command, who did not undergo or receive any rehabilitation therapy, who received any surgical intervention or botulinum toxin injection within the last 3 months prior to the study, or who had received home-based private physiotherapy. Children who received recent ( 3 months or less) botulinum toxin and surgery were also excluded as these interventions may reduce their muscle strength and hence influence the outcomes of this study. Parents or main caregivers were given an explanation of the study procedure and SHEP, as well as on their rights to withdraw their children from participating in the research, before they provided the written informed consent.

A total of 38 ambulant children with $\mathrm{CP}$ were shortlisted from the clinic registry on the basis of the inclusion criteria but had further reduced to 14 after the exclusion criteria were considered. Of the fourteen remaining children, two were removed because their parents did not provide the consent due to inability to comply for repeated assessments and their negative attitude towards exercise.

\subsection{Procedure}

The SHEP provides a general, structured and written exercise prescription particularly for ambulant children with CP. Standard care provides an individualised programme for a particular child's needs, and may vary from one centre to another. SHEP is a general, practical, and written home-based exercise programme added to standard care, which may guide all ambulant children with $\mathrm{CP}$ to execute a scheduled exercise at home under the supervision of parents or caregivers.

The SHEP protocol is for ambulant children with $\mathrm{CP}$ and consisted of the following three main exercise components for the upper and lower limb muscles: aerobic fitness training, muscle strengthening, and stretching exercises. These components conform to the American College of Sports Medicine guidelines for persons with chronic disease and disabilities [16].

Aerobic exercise for the upper and lower limbs involved throwing-and-catching ball activities and step exercises (with forward, backward and side-to-side movement). A play ball $20 \mathrm{~cm}$ in diameter and a stepping stool at $18 \mathrm{~cm}$ in height were provided to all participants.

Muscle strengthening exercises were performed using a resistive band or a $500 \mathrm{~g}$ weighted strap. The exercise programme can be performed in lying, sitting, or standing postures depending on the muscle involved and the ability of the participant to perform the specific movement. Specific exercises included repetitive movements using the weighted strap or resistive bands placed at the wrist and ankle of both limbs. Movements involve bilateral shoulder abduction and flexion, elbow extension and flexion, and hip extension and abduction, as well as knee extension and flexion.

Stretching exercise was emphasised before every aerobic and strengthening exercise in the SHEP. It involved stretching of all joints of bilateral upper and lower limbs to avoid musculoskeletal injuries during exercise.

The OMNI rate of perceived exertion (OMNI-RPE) was used as a tool for the self-perceived assessment of physical exertion to guide the progression of exercise intensity, which is individually varied [17]. The feasibility of the OMNI-RPE for the CP population has been previously validated and is strongly associated with the physiological biomarkers of aerobic ex- 


\begin{tabular}{|c|c|c|c|c|c|c|c|c|}
\hline \multicolumn{2}{|c|}{ Week: 8} & \multicolumn{7}{|c|}{ Date: } \\
\hline \multirow{2}{*}{\multicolumn{2}{|c|}{ EXERCISES }} & \multicolumn{7}{|c|}{ DAY } \\
\hline & & 1 & 2 & 3 & 4 & 5 & 6 & 7 \\
\hline 1 & STRETCHING & & & & & & & \\
\hline 2 & $\begin{array}{l}\text { AEROBIC } \\
\text { Upper limb (UL) } \\
\text { Lower limb (LL) }\end{array}$ & $\begin{array}{l}\text { OMNI- } \\
\text { RPE: } \\
\text { Min: } \\
\text { Min: }\end{array}$ & & & & $\begin{array}{l}\text { OMNI- } \\
\text { RPE: } \\
\text { Min: } \\
\text { Min: }\end{array}$ & & \\
\hline 3 & $\begin{array}{l}\text { STRENGTHENING } \\
\text { Upper limb (UL) } \\
\text { Lower limb (LL) }\end{array}$ & & & $\begin{array}{l}\text { OMNI- } \\
\text { RPE: } \\
\text { Rep: } \\
\text { Rep: }\end{array}$ & & & & $\begin{array}{l}\text { OMNI- } \\
\text { RPE: } \\
\text { Rep: } \\
\text { Rep: }\end{array}$ \\
\hline
\end{tabular}

Fig. 2. Example of SHEP schedule.

ercise intensity (e.g., heart rate [HR] and $\mathrm{VO}_{2}$ ) [18]. During strengthening and aerobic exercises, participants were advised to achieve an OMNI-RPE value between 5 and 8 as a guide to their activity threshold for moderate intensity training, as required to improve cardiorespiratory fitness [19]. Participants and caregivers were advised not to exceed an OMNI-RPE value of 8 to avoid any complications, particularly musculoskeletal injuries. The OMNI-RPE tool was introduced to the participants during the familiarisation session in Phase 1.

During SHEP, the caregiver monitored the participants and recorded the details of each exercise modality in the given logbook. The duration of both aerobic and strengthening activities depended on how long (in minutes) each participant was able to execute every type of exercise until reaching the OMNI-RPE value of 5-8. The frequency of exercise was three times per week for the first until fourth week and increased to four times per week on the fifth until eighth week of the intervention, with alternating aerobic and strengthening components (Fig. 2). The frequency of exercise was increased gradually in order to provide progression in the exercise programme. The logbook also included written and pictorial instruction describing the SHEP intervention.

\subsection{Outcomes}

Walking distance, resting time, and final HR were recorded during a 6 minute walk test (6MWT). Time was recorded using a stopwatch as the participant walked from one point to another, with a turn at the endpoints. A basketball court was used as a venue for the 6MWT assessment. A meter tracker was used to accurately determine the walking distance. The resting HR before the 6MWT and the highest observed at the end of 6 minutes were recorded using a chest-worn HR monitor for children (POLAR FT 60 model, PLR-FT$60 \mathrm{~F})$.

The primary outcome included the walking speed (meter per minute), which was derived by dividing the distance (meter) by $6 \mathrm{~min}$. Speed (meter per minute) was used as the denominator to calculate the HR difference (final HR at 6 minutes - resting HR; beats per minute) for measuring the physiological cost index (PCI), which was expressed as beats per meter. High PCI values indicate a low walking efficiency (i.e., higher HRs at slower gait speeds), and the PCI in children with $\mathrm{CP}$ is six times greater than that in normal children [20].

GMFM-66 comprised 66 motor functions, which included dimensions A, B, C, D, and E. Dimensions D and $\mathrm{E}$ were selected to assess the standing, walking, running, and jumping domains [21]. GMFM results were converted into percentages. GMFM-66 has high sensitivity for motor function changes in the CP population and has been used in studies on CP interventions [22].

\subsection{Statistical analysis}

All data were analysed using the statistical package SPSS version 23. The repeated measurements obtained on a given individual within a phase were averaged. These averaged measurements were then compared across phases by repeated measures of analysis of variance (RM ANOVA) to analyse the effect of 
Table 1

Descriptive data of walking distance and speed $(n=11)$

\begin{tabular}{lcr}
\hline Outcome & Mean & SD \\
\hline 6MWT & & \\
Distance (m) & & \\
$\quad$ Phase 1 & 334 & 11.3 \\
Phase 2 & 381 & 30.1 \\
Phase 3 & 378 & 1.9 \\
Speed (m·min -1 ) & & \\
$\quad$ Phase 1 & 56.0 & 4.6 \\
Phase 2 & 63.4 & 4.4 \\
Phase 3 & 62.9 & 4.4 \\
\hline
\end{tabular}

intervention within the same participant at the three SHEP phases. Distribution normality was assessed using the Shapiro-Wilk test. Non-normally distributed data were analysed using the Wilcoxon signed-rank test, and all pairwise comparisons were adjusted for multiple comparisons by using an adjusted significance level of $0.05 / 3=0.017$. Effect sizes were calculated by obtaining the partial $\eta$-squared value, which was derived from RM ANOVA and then converted into Cohen's $d$. The $t$-statistic (df) value of descriptive data were used to calculate the effect size and Cohen's $d$ for non-normally distributed data [23].

\section{Results}

Twelve participants were recruited, but one dropped out after completing Phase 1 due to inability to commit for further multiple outcome assessments. Thus, 11 children fully participated in this study (7 males and 4 females, mean age 10 years and 3 months, standard deviation (SD) 3 years). The majority of the study participants were classified as hemiplegic $\mathrm{CP}(n=8)$, primary school aged, 6-12 years old $(n=8)$ and male $(n=7)$. The participants were functionally classified as GMFCS I $(n=7)$, GMFCS II $(n=3)$ and GMFCS III $(n=1)$. Outcomes were measured by means and median IQR for each individual accordingly; with weekly intervals over 4 weeks in Phase 1, three measurements over 8 weeks in Phase 2 and two measurements in Phase 3, at the first and third month after intervention.

The descriptive data for each outcome are presented in Tables 1 and 2. For each individual, data for walking distance and speed were averaged within each phase. Over the 6MWT, their average walking distance increased by $47 \mathrm{~m}$ after the SHEP intervention in Phase 2 and was maintained during followup. Then, walking distance further increased by $12.3 \%$
Table 2

Descriptive data on PCI and GMFM$66(n=11)$

\begin{tabular}{lcc}
\hline Outcome & Median & IQR \\
\hline PCI (beat $\cdot \mathrm{m}^{-1}$ ) & & \\
Phase 1 & 0.56 & 0.77 \\
Phase 2 & 0.55 & 0.63 \\
Phase 3 & 0.47 & 0.57 \\
GMFM-66 & & \\
Phase 1 & 91.88 & 17.4 \\
Phase 2 & 93.38 & 16.3 \\
Phase 3 & 95.25 & 17.5 \\
\hline
\end{tabular}

and speed increased by $11.7 \%$ in Phase 2; both maintained above the initial value in Phase 3. Distance was measured in meters and normally distributed across all phases. Overall, a difference was found in walking distance across phases $(p=0.001)$. Further pairwise comparison revealed a significant difference from Phase 1 to Phase 2, whereby the walking speed was 7.4 units lower during Phase 1 compared with that during Phase 2 (95\% confidence interval $[\mathrm{CI}],-11.8$ to -3.1 ), and the walking distance was 46.7 meters lower in Phase 1 compared with that in Phase 2 (95\% CI, -67.0 to -26.4 ) (Table 3). The RM ANOVA on 6 min walking distance and speed demonstrated that the home-based intervention significantly increased walking speed, with the partial $\eta$-squared of $p=0.01$, calculated effect size of 0.488 , and Cohen's $d$ of 1.9.

PCI and GMFM-66 results were analysed through a Wilcoxon signed-rank test by using an adjusted significance level at $0.05 / 3=0.017$ due to non-normally distributed data (as previously described). PCI results demonstrated a slightly improved walking efficiency between the Phase 2 and Phase 3 assessments $(t$ statistic $=-2.103)$ (Table 4). However, this finding was not statistically significant and displayed a small effect size (Cohen's $d=0.39$ ). As shown in Table 4, most positive changes in the domains $\mathrm{D}$ and $\mathrm{E}$ for GMFM-66 score were statistically significant $(p<$ 0.017). The improvement of GMFM-66 scores during Phase 2 and Phase 3 had a large effect size, with Cohen's $d$ of 1.039 and 1.054, respectively, compared with that during Phase 1.

The returned logbook documentation $(n=7)$ indicated that the maximum mean duration of exercise for each session was 23.5 minutes recorded on the seventh week, whereas the minimum mean duration was 16.9 minutes documented during the second week. This result reflected a $28 \%$ increase in mean duration over 5 weeks of SHEP. The information from the logbook also provides an overall picture of the ability of the participants to gradually increase their exer- 
Table 3

Changes of walking distance and speed $(n=11)$

\begin{tabular}{|c|c|c|c|c|c|}
\hline \multirow[t]{2}{*}{ Comparison } & \multirow[t]{2}{*}{ Mean difference } & \multirow[t]{2}{*}{ Std. error } & \multirow[t]{2}{*}{ Sig. } & \multicolumn{2}{|c|}{$95 \%$ confidence interval for difference } \\
\hline & & & & Lower bound & Upper bound \\
\hline \multicolumn{6}{|c|}{ Six minutes' walk distance } \\
\hline Phase 1-Phase 2 & -46.7 & 9.1 & 0.000 & -67.0 & -26.4 \\
\hline Phase 2-Phase 3 & -1.5 & 9.2 & 0.878 & -22.3 & 19.4 \\
\hline Phase 3-Phase 1 & 48.29 & 15.3 & 0.012 & 13.7 & 82.9 \\
\hline \multicolumn{6}{|c|}{ Average walking speed } \\
\hline Phase 1-Phase 2 & -7.4 & 1.5 & 0.002 & -11.8 & -3.1 \\
\hline Phase 2-Phase 3 & 0.5 & 1.6 & 1.000 & -4.0 & 5.0 \\
\hline Phase 3-Phase 1 & 7.0 & 2.5 & 0.056 & -0.2 & 14.1 \\
\hline
\end{tabular}

Six minute walk distance expressed in $\mathrm{m}$. Average walking speed expressed in $\mathrm{m} \cdot \mathrm{min}^{-1}$.

Table 4

\begin{tabular}{lcccc}
\multicolumn{6}{c}{ Comparison of PCI and GMFM between different phases $(n=11)$} \\
\hline Outcome & Median (IQR) & Median (IQR) & $t$-statistic (df) & $p$-value \\
\hline PCI & & & & \\
Phase 1-Phase 2 & $0.56(0.77)$ & $0.55(0.63)$ & -1.313 & 0.19 \\
Phase 2-Phase 3 & $0.55(0.63)$ & $0.47(0.57)$ & -2.103 & 0.03 \\
Phase 3-Phase 1 & $0.47(0.57)$ & $0.56(0.77)$ & -1.130 & 0.26 \\
GMFM-66 & & & & \\
Phase 1-Phase 2 & $91.88(17.4)$ & $93.38(16.3)$ & -3.451 & 0.001 \\
Phase 2-Phase 3 & $93.38(16.3)$ & $95.25(17.5)$ & -2.621 & 0.009 \\
Phase 3-Phase 1 & $95.25(17.5)$ & $91.88(17.4)$ & -3.502 & 0.000 \\
\hline
\end{tabular}

Note: Wilcoxon signed-rank test. Adjusted statistical analysis due to non-normal distribution, as described in text. PCI expressed in beat $\cdot \mathrm{m}^{-1}$. GMFM expressed as percentage $(\%)$.

cise duration and achieve the anaerobic threshold, with OMNI-RPE value of 5-7 in aerobic and strengthening exercise components.

Overall, these results showed the positive effect of SHEP on the walking distance, speed and GMFM66 score of the study participants. These observations were particularly distinctive between Phase 1 (preintervention) and Phase 2 (intervention) assessments. However, a larger magnitude of intervention effect on walking speed was observed in the effect size analysis compared with that in the GMFM-66.

\section{Discussion}

This study revealed that walking speed and GMFM66 (components D \& E) significantly improved after SHEP intervention. However, the improvement of walking speed was not sustained during follow-up (Phase 3). Bania et al. concluded that although exercise programmes and online support programmes can increase habitual physical activity in people with $\mathrm{CP}$, these effects are not maintained when the programmes are terminated [5]. Similarly, a study involving an 8 month group exercise training programme with GMFCS I-II participants found linear positive effects on aerobic fitness, anaerobic capacity, agility, muscle strength and GMFM throughout the intervention, but these positive outcomes on dimensions of physical fitness had decreased 4 months after the intervention was terminated [6].

The exercise tolerance of the participants in this study improved as they progressed through the intervention. At the end of the study, participants reported a mean exercise duration of 23.5 minutes per session, higher than their mean duration of 16.9 minutes when they first commenced the home-based programme. Nevertheless, the SHEP is a program composed of alternating aerobic and strengthening exercises program, which may not exactly conform to the current exercise recommendation for children with $\mathrm{CP}$ where a minimum of 20 minutes per session of aerobic exercise is recommended [24].

Walking speed is an objective measure of walking ability and may reflect the general function and quality of life in children with CP [25,26]. Deterioration in walking speed might indicate a decline in a child's daily activities, thereby limiting their interaction and participation in society and ultimately affecting their quality of life $[27,28]$. Self-paced walking speed was selected by performing 6MWT, which is a reliable measure of walking ability for the CP population [29]. 
PCI represents a proxy of cardiovascular stress and has been deployed as a measure of the walking efficiency in children with CP [20]. Given that HR is affected by walking speed, the PCI, which combines the two parameters, can be a useful parameter to assess walking efficiency. A more accurate method of measuring energy cost of walking in children with $\mathrm{CP}$ would entail directly measuring their oxygen consumption and then normalising this by their average walking speed [30]. However, PCI was selected because it is practical and requires low-cost equipment to monitor in daily clinical practice. The findings from this current study revealed that PCI decreased in Phase 2 assessment relative to baseline (Phase 1). Nevertheless, even though exercise intervention improved walking speed, it did not necessarily exert the same significant effect on walking efficiency. Another measure of the effects of the intervention is HR recovery or HR variability, which directly quantifies cardiovascular physiological stresses due to the association with physical fitness, exercise intensity, and autonomic function [31].

SHEP allowed parents and children to monitor the intensity of each exercise modality on the basis of OMNI-RPE; an OMNI-RPE value between 5-8 indicates at least a moderate intensity level of exercise [19]. OMNI-RPE was selected because it is more feasible, reliable and practical than HR monitoring when performed at home under caregiver supervision. Moderate intensity training is suggested, given that children with CP are capable of and will benefit from engaging in progressively intense aerobic exercise similar to the extent recommended for their peers with typical development [24].

The findings from our study suggest that exercise is an important daily physical activity that improves the walking ability of ambulant children with CP. A recently published guideline for exercise and physical activity recommended that healthy lifestyle and physical activity should be encouraged and added as part of therapy for children with $\mathrm{CP}$ as they transition into adulthood [24]. Therefore, in the clinical context, SHEP can serve as an introductory tool to physical activity for young people with $\mathrm{CP}$ and may be potentially used as a structured and rapid exercise intervention to improve walking ability, particularly walking speed, for a short period of 8 weeks when added to standard care. SHEP is also an example of an active training protocol, which is recommended as a homebased exercise for the rehabilitation of individuals with CP [4].
This study had several limitations. Firstly, the participants were familiarised with SHEP during Phase 1. Even though familiarisation was conducted before the intervention (when all participants had been given their SHEP logbook), some of the learning and motivation effects of SHEP may have already been embedded to amplify positive effects on outcomes. A separate familiarisation session for SHEP should be instituted prior to Phase 1. Secondly, HR was only measured at rest and at the end of 6MWT. The average HR obtained during walking only reflected the actual walking efficiency during the 6MWT. Another important limitation in this study was the small number of participants, thereby limiting the number of participants in each GMFCS I-III group, as well as in different age groups; hence, further subanalysis to look into differences between age groups was not appropriate. Given that only one GMFCS III participant was included, the results might not be fully representative of the entire group of ambulant children with CP from GMFCS IIII and across all ages. SHEP is also unsuitable for children severely affected with $\mathrm{CP}$, particularly those in GMFCS IV and V. SHEP done by participants at home was monitored with a logbook. Parents or caregivers used the logbook as a guide and to record SHEP activities at home. Though the logbook was a simple and feasible way to monitor and follow-up participants, its primary limitation is the lack of reliability and validity of the recorded activities.

\section{Conclusion}

SHEP was implemented in accordance with the exercise recommendation for CP [24]. It is a potential, practical, and structured home-based exercise programme that can improve the walking function of children with ambulant CP. Although its effect was not significantly sustained after the programme, it can be a useful tool as a structured and rapid exercise intervention to achieve a short term goal when added to standard care. A large-scale RCT with a large group of participants with ambulant CP can enable additional meaningful results to be obtained for generalisation.

\section{Acknowledgments}

The authors thank the staff of the Paediatric Rehabilitation Unit and Rehabilitation Clinic in the University Malaya Medical Centre for their assistance in 
this study. This study was supported and funded by the University Malaya Research Fund Assistance Grant (BK029-2015) and registered with the Australian New Zealand Clinical Trial Registry (ANZCTR); registration number ACTRN12616001013459.

\section{Conflict of interest}

The authors have no conflicts of interest to disclose.

\section{References}

[1] Aisen ML, Kerkovich D, Mast J, Mulroy S, Wren TA, Kay RM, et al. Cerebral palsy: Clinical care and neurological rehabilitation. Lancet Neurol. 2011; 10: 844-52. doi: 10.1016/ S1474-4422(11)70176-4.

[2] Rosenbaum P, Paneth N, Leviton A, Goldstein M, Bax M, Damiano D, et al. A report: The definition and classification of cerebral palsy. Dev Med Child Neurol. 2007; 109: 8-14. doi: 10.1111/j.1469-8749.2007.tb12610.x.

[3] Kerr Graham H, Selber P. Musculoskeletal aspects of cerebral palsy. J Bone Joint Surg Br. 2003; 85(2): 157-66. doi: 10.1302/0301-620X.85B2.14066.

[4] Damiano DL. Activity, activity, activity: Rethinking our physical therapy approach to cerebral palsy. Phys Ther. 2006; 86 : 1534-40. doi: 10.2522/ptj.20050397.

[5] Bania T, Dodd KJ, Taylor N. Habitual physical activity can be increased in people with cerebral palsy: A systematic review. Clin Rehabil. 2011; 24(4): 303-15. doi: 10.1177/0269 215510383062.

[6] Verschuren O, Ketelaar M, Gorter JW, Helders PJ, Uiterwaal CS, Takken T. Exercise training program in children and adolescents with cerebral palsy: A randomized controlled trial. Arch Pediatr Adolesc Med. 2007; 161(11): 1075-81.

[7] Novak I, McIntyre S, Morgan C, Campbell L, Dark L, Morton $\mathrm{N}$, et al. A systematic review of interventions for children with cerebral palsy: State of the evidence. Dev Med Child Neurol. 2013; 55(10): 885-910. doi: 10.1111/dmcn.12246.

[8] Majnemer A, Shevell M, Law M, Poulin C, Rosenbaum P. Indicators of distress in families of children with cerebral palsy. Disabil Rehabil. 2012; 34(14): 1202-7. doi: 10.3109/09638 288.2011.638035.

[9] Verschuren O, Wiart L, Hermans D, Ketelaar M. Identification of facilitators and barriers to physical activity in children and adolescents with cerebral palsy. J Pediatr. 2012; 161(3): 48894. doi: 10.1016/j.jpeds.2012.02.042.

[10] Novak I, Cusick A. Home programmes in paediatric occupational therapy for children with cerebral palsy: Where to start? Aust Occup Ther J. 2006; 53: 251-64. doi: 10.1111/j.14401630.2006.00577.x

[11] Tinderholt Myrhaug H, Østensjø S, Larun L, Odgaard-Jensen $\mathrm{J}$, Jahnsen R. Intensive training of motor function and functional skills among young children with cerebral palsy: A systematic review and meta-analysis. BMC Pediatr. 2014; 14: 1. doi: 10.1186/s12887-014-0292-5.

[12] Jeng SC, Yeh KK, Liu WY, Huang WP, Chuang YF, Wong AMK, et al. A physical fitness follow-up in children with cerebral palsy receiving 12 -week individualized exercise training. Res Dev Disabil. 2013; 34(11): 4017-24. doi: 10.1016/j.ridd. 2013.08.032.
[13] Johnston TE, Watson KE, Ross SA, Gates PE, Gaughan JP, Lauer RT, et al. Effects of a supported speed treadmill training exercise program on impairment and function for children with cerebral palsy. Dev Med Child Neurol. 2011; 53(8): 74250. doi: 10.1111/j.1469-8749.2011.03990.x.

[14] Lorentzen J, Greve LZ, Kliim-Due M, Rasmussen B, Bilde PE, Nielsen JB. Twenty weeks of home-based interactive training of children with cerebral palsy improves functional abilities. BMC Neurol. 2015; 15: 75. doi: 10.1186/s12883015-0334-0.

[15] Van Wely L, Balemans ACJ, Becher JG, Dallmeijer AJ. Physical activity stimulation program for children with cerebral palsy did not improve physical activity: A randomised trial. J Physiother. 2014; 60(1): 40-9. doi: 10.1016/j.jphys. 2013.12.007.

[16] Maltais DB. Cerebral palsy. In: ACSM's Exercise Management for Persons with Chronic Diseases and Disabilities. $4^{\text {Th }}$ ed. Moore G, Durstine JL, Painter P, Editors. United States of America: Human Kinetics. 2016; 256-266.

[17] Utter AC, Robertson RJ, Nieman DC, Kang J. Children's OMNI scale of perceived exertion: Walking/running evaluation. Med Sci Sports Exerc. 2002; 34(1): 139-44.

[18] Fragala-Pinkham M, O'Neil ME, Lennon N, Forman JL, Trost SG. Validity of the OMNI rating of perceived exertion scale for children and adolescents with cerebral palsy. Dev Med Child Neurol. 2015; 57(8): 748-53. doi: 10.1111/dmcn.12703.

[19] Robertson RJ, Goss FL, Aaron DJ, Utter AC, Nagle E. Omni scale rating of perceived exertion at ventilatory breakpoint by direct observation of children's kinematics. Percept Mot Skills. 2007; 104(3): 975-84.

[20] Raja K, Joseph B, Benjamin S, Minocha V, Rana B. Physiological cost index in cerebral palsy: Its role in evaluating the efficiency of ambulation. J Pediatr Orthop. 2007; 27(2): 1306. doi: 10.1097/01.bpb.0000242440.96434.26.

[21] Russell DJ, Rosenbaum PL, Wright M, Avery LM. Gross motor function measure (GMFM-66 and GMFM-88). User's Manual: Wiley Blackwell Publishing. 2013.

[22] Alotaibi M, Long T, Kennedy E, Bavishi S. The efficacy of GMFM-88 and GMFM-66 to detect changes in gross motor function in children with cerebral palsy (CP): A literature review. Disabil Rehabil. 2014; 36(8): 617-27. doi: 10.3109/09638288.2013.805820.

[23] Sulllivan MG, Feinn R. Using effect size - or why the p value is not enough. J Grad Med Educ. 2012; 279-82. doi: 10.4300/JGME-D-12-00156.1

[24] Verschuren O, Peterson MD, Balemans AC, Hurvitz EA. Exercise and physical activity recommendations for people with cerebral palsy. Dev Med Child Neurol. 2016; 58(8): 798-808. doi: 10.1111/dmcn.13053.

[25] Pirpiris M, Wilkinson AJ, Rodda J, Nguyen TC, Baker RJ, Nattrass GR, Graham HK. Walking speed in children and young adults with neuromuscular disease: Comparison between two assessment methods. J Pediatr Orthop. 2003; 23: 302-307.

[26] Pirpiris M, Gates PE, McCarthy JJ, D’Astous J, Tylkowksi $\mathrm{C}$, Sanders JO, et al. Function and well-being in ambulatory children with cerebral palsy. J Pediatr Orthop. 2006; 26: 119124.

[27] Schenker R, Coster W, Parush S. Participation and activity performance of students with cerebral palsy within the school environment. Disabil Rehabil. 2005; 27: 539-552. doi: 10.1080/09638280400018437. 
[28] Beckung E, Hagberg G. Neuroimpairments, activity limitations, and participation restrictions in children with cerebral palsy. Dev Med Child Neurol. 2002; 44: 309-316.

[29] Maher CA, Williams MT, Olds TS. The six-minute walk test for children with cerebral palsy. Int J Rehabil Res. 2008; 31(2): 185-8. doi: 10.1097/MRR.0b013e32830150f9.

[30] Kamp FA, Lennon N, Holmes L, Dallmeijer AJ, Henley J, Miller F. Energy cost of walking in children with spastic cerebral palsy: Relationship with age, body composition and mo- bility capacity. Gait Posture. 2014; 40(1): 209-14. doi: 10 1016/j.gaitpost.2014.03.187.

[31] Cornelissen VA, Verheyden B, Aubert AE, Fagard RH. Effects of aerobic training intensity on resting, exercise and postexercise blood pressure, heart rate and heart-rate variability. J Hum Hypertens. 2010; 24(3): 175-82. doi: 10.1038/jhh. 2009.51. 